\title{
Technological support for the enactment of collaborative scripted learning activities across multiple spatial locations
}

\author{
Luis de-la-Fuente-Valentín ${ }^{1,}$ *, Mar Pérez-Sanagustín ${ }^{2,}$ **, Davinia Hernández-Leo 3 , \\ Abelardo Pardo ${ }^{4}$, Josep Blat ${ }^{3}$, Carlos Delgado Kloos ${ }^{2}$ \\ ${ }_{1}$ Research \& Technology, International University of La Rioja, Paseo de la Castellana 163, Madrid, Spain, 2 Department of Telematic Engineering, University \\ Carlos III of Madrid, Av. Universidad, 30, 28911, Leganés (Madrid), Spain, s Department of Information and Communication Technologies, Universitat \\ Pompeu Fabra, Barcelona, Spain \\ ${ }_{4}$ School of Electrical and Information Engineering, The University of Sydney, 2006 NSW, Australia \\ * Corresponding author. Tel.: +34 91 5674391. ** Corresponding author. Tel.: +34 916248437
}

E-mail addresses: luis.delafuente@unir.net, luis.fuente.valentin@gmail.com (L. de-la-Fuente-Valentín), mmpsanag@it.uc3m.es (M. Pérez-Sanagustín), davinia.hernandez@upf.edu (D. Hernández-Leo), abelardo.pardo@sydney.edu.au (A. Pardo), josep.blat@upf.edu (J. Blat), cdk@it.uc3m.es (C. Delgado Kloos).

\begin{abstract}
Computer Supported Collaborative Blended Learning (CSCBL) scripts are innovative practices that benefit from interactive devices to combine and coordinate activities occurring in different spatial locations. However, the adoption of CSCBL scripts is hindered by the difficulties in orchestration that they entail for practitioners. As observed in a concrete experiment, these orchestration problems include: adapting group formation according to students' actions in previous activities, supporting transitions between activities and artifacts across locations using diverse technologies, or displaying the appropriate tools to students depending on their group and assigned task. This paper describes the technological support designed to alleviate this complexity. The result is a Computer Supported Collaborative Blended Learning (CSCBL) script and its associated orchestration system that allows the replication of these practices at a minimum cost. The CSCBL script has been evaluated in a case study with 35 students and 5 teachers. Based on this orchestration system and the findings of the experiment, we also propose an architecture based on IMS Learning Design and Generic Service Integration in combination with other web based tools to support the enactment of other similar CSCBL scripts. The findings of this experiment offer interesting insights into the extend to which different technologies and multiple spaces can be combined for orchestrating integrated complex collaborative practices.
\end{abstract}

Keywords: CSCL scripts Orchestration, IMS Learning Design Service integration, Case study

\section{Introduction}

In the last decade, the introduction of interactive and portable devices in education has prompted the appearance of new opportunities for collaborative learning [1-4]. Works such as the one proposed by [5], for example, show how mobile computers can be used as a guide for supporting environmental learning with motivational benefits for the students. Also, [6] demonstrates how mobile phones promote active learning and can help students to understand animal behavior in the savannah.
These studies benefit from the connectivity of mobile and portable technologies to create collaborative learning scenarios in which activities occurring at different spatial locations, in and beyond the classroom, are combined, structured and integrated into a unique learning flow. We define these innovative situations as Collaborative Blended Learning (CBL) practices.

One of the major difficulties of CBL practices when enacted in the actual educational context relies on coordinating and 
monitoring the different activities so as to produce effective collaboration. In the field of Computer Supported Collaborative Learning (CSCL), some authors support the idea that collaborative processes have to be orchestrated. Orchestration stands for the coordination and management of a whole learning group in order to maintain the process towards the learning outcomes $[7,8]$. Practitioners orchestrate their activities in different dimensions in order to achieve certain goals: in a social dimension (individual, group or whole-class activities), in a pedagogical dimension (which implies the adaptation of the designed activities to the occurrences of the classroom), and in a technological dimension (coordination of the transactions among software components) [9].

One of the most well-known forms of coordinating these dimensions is the use of scripts. The rationale of the scripts is to structure collaborative learning processes in order to trigger group interactions that may be rare in free collaboration $[10,11]$. When these interactions are technologically mediated, they are called Computer Supported Collaborative Learning scripts (or CSCL scripts) [8]. In this paper, and by analogy with CSCL scripts, we refer to CSCBL scripts as the mean for coordinating a CBL practice. Therefore, CSCBL scripts are, essentially, a more distributed form of CSCL scripts for supporting the coordination of collaborative practices that combine formal and informal activities occurring across different spatial locations [12].

Several studies show the effectiveness of CSCL scripts to support collaborative learning in certain educational scenarios. For instance, the Universanté script has been used in the health teaching community for medical students from different countries $[13,14]$. This script is structured around phases and roles in which activities rely on the confrontation of different national health countries by exploiting the socio-economic and cultural differences between countries. Another example is the ArgueGraph script, which is aimed at triggering discussion between pairs of students with heterogeneous opinions [15].

However, and despite the effectiveness of these solutions for supporting CSCL scripts, none of these solutions is able to capture the complex orchestration requirements of CSCBL scripts. In the pedagogical dimension, the adaptation of the designed activities in CSCBL scripts implies tracking the students' progress in and beyond the classroom and managing the learning flow according to the students' performance [16]. In the technological dimension, CSCBL scripts require practitioners to integrate and combine different technologies in order to assure the interrelation between activities from a data flow perspective and facilitating the management of the learning flow.

All these orchestration requirements were clearly identified in a previous work [17] in which a CSCBL script called "Discovering the campus together (2009 Edition)" was proposed. The result was an experience that combined outdoors activities technologically supported by mobile phones and in-class collaborative sessions for facilitating first-engineering students learn about the campus and its services. The script was enacted with 74 students at the University Pompeu Fabra with encouraging results in terms of learning benefits. However, the results also showed that the technological support proposed for the script enactment imposed a significant workload to the teaching staff. This workload, mostly related to management tasks, poses a limit on the scalability and reusability of the enacted activities and suggests the use of a method for the automation of certain orchestration tasks.

This paper takes as a basis the limitations detected in this previous study to propose a new technological orchestration system that aims to solve them. The result is a new version of the CSCBL script "Discovering the campus together (2010 Edition)". Both editions share lot of similarities because they were designed according to a model called 4SPPIces. 4SPPIces is a conceptual model that provides practitioners and technicians with a common language to design CSCBL scripts and the technological setting for supporting their enactment [12]. However, the implementation of the scripts in each edition differ on the technology employed to support their enactment. The second version of the script was technologically supported with the use of: (1) IMS Learning Design (IMS LD) to computationally represent the learning flow, (2) Google Spreadsheets to manage the students' groups and (3) Generic System Integration to integrate all these management tasks with the mobile-phone-supported outdoors activity, all combined with the use of mobile phones and Near Field Communication (NFC, [18]) technologies. The combination of IMS LD and case specific tools represents the basis of an underlying architecture for the support of CSCBL scripts. In order to validate the proposal and evaluate whether it maintains the educational benefits observed in the first implementation of the first edition of the script we present an experiment which, on the one hand, evaluates if the proposed system satisfies the requirements and limitations of the script and, on the other hand, allows an exploratory analysis of the CSCBL situation. In the experiment, the new CSCBL script is enacted with the participation of 35 freshmen students of an engineering degree of the Universitat Pompeu Fabra, Barcelona. The data collected in the experiment is analyzed and triangulated following a mixed methodology.

The structure of this paper is as follows: first, Section 2 gives an overview of the previous edition activity flow, highlighting the benefits and drawbacks encountered during its enactment. Next, Section 3 depicts the proposed orchestration system, details how the different technologies were combined to support the whole learning flow, describes how the learning flow is codified using IMS LD, sets the basis of the underlying architecture, and finishes describing the design process of the script using the 4SPPIces model. Then, Section 4 presents the case study by describing the context in which the script was enacted, the evaluation methodology used to analyze the data extracted from the experiment and the main results. Finally, Section 5 draws conclusions from this work and discusses the validity of the proposed orchestration system. We contend that both the particular case presented and the derived underlying architecture are valuable contributions for inspiring other researchers in the deployment of innovative scripted collaborative learning activities in multiple spacial locations.

\section{Description of the problem}

This section describes the enactment of the previously deployed experiment, called "Discovering the campus together (2009 Edition)", and reports on the orchestration problems detected. This script was structured according to the Jigsaw Collaborative Learning Flow Pattern (CLFP). CLFPs aim at capturing the essence of well-known techniques for structuring a flow of learning activities to potentially produce effective learning from collaborative situations [19]. This CLFP intends: (1) to foster collaboration amongst students so they can meet each other and (2) to integrate the set of activities into a coherent flow of learning activities. The details about the enactment of this first script version are reported in [17], while the results of the orchestration problems analysis have been collected in [20]. Both, the enactment of the experiment and its analysis lay the foundations for proposing a new CSCBL script and its associated technological system for supporting its enactment to solve these limitations while granting the same learning outcomes.

\subsection{The enacted activity flow: 2009 edition}

The activity "Discovering the campus together (2009 Edition)" was proposed to help fresh engineering students in their first 
contact with the campus, its services, and the university community, methodologies and activities. The activity was structured as a sequence of activities with three different phases:

- "Discovering the campus". This activity consisted in an individual campus exploration. Students had 30 min to freely walk around the campus and access the information stored in 46 NFC tags previously distributed by the teachers across the 5 different buildings of the campus. These tags contained information about their location. Students were equipped with NOKIA (N6131, N6212) mobile phones with an embedded RFID (Radio-frequency identification, [21]) reader to access the information stored in the tags. All the information regarding the sequence of tags accessed by each student was stored into a $\log$ file in order to capture the students' routes. After the visit, students had to fill in a Google Forms questionnaire indicating which buildings they had visited and which seemed to them the most interesting.

The task for the teachers in this phase was: (1) to annotate the time when the device was given to a student in order to identify which log file belonged to each student, (2) to store the students' log files in the computer via a Bluetooth connection and (3) to associate each log to each student according to the notes about the people participating and the times taken and the end of the exploration.

- "Explain the campus". In this phase, students were grouped into "Building Expert groups". Each group was assigned to a building by the teacher and had to create a slide-based presentation explaining the main characteristics of their building. Once finished, they had to upload the presentation to the Moodle Platform of the University (henceforth Moodle).

The teacher formed the groups by manually bringing together 4 or 5 students with a similar building expertise level. This expertise level was defined considering two sources of information: (1) the log files obtained during the exploration and (2) the answers to the Google Forms questionnaire. For instance, the experts on building A were those students that visited a higher number of tags located in $A$ and that preferred that building in their answers to the questionnaire. This phase required the collaboration of the different students in the group in order to produce a presentation according to the information extracted by each group member during the first phase. Forming expert groups according to the students' expertise and making them to work and discuss on the final presentation is been shown as a good mechanism to encourage student participation $[19,17]$.

- "Reflect about the campus". Students had to access and review all the presentations uploaded to Moodle to obtain the information necessary for answering an individual test including questions about the whole campus. This final test took 25 min in a classroom session with PCs.

The teachers task in this phase was to upload all the delivered presentations into a Moodle-based public repository and prepare the final questionnaire with the information included in the delivered presentations.

The activity entailed several motivational and learning benefits for the students. The students learned about the campus services, got familiar with some of the academic methods typically employed in their studies and had a first contact with colleagues from their courses. Also, the results of this experiment indicate that the script was also a good mechanism to enhance and support collaboration. The students had a first collaborative experience working with colleagues from different degrees. At the same time, the use of technologies such as mobile phones motivated the students and fostered their interest in technology.

\subsection{Detected limitations}

All the management tasks required for the script orchestration were carried out by two teachers and one researcher. The activity was technologically supported (NFC tags, mobile phones, Moodle) but there was no system that automatically integrated the whole process. This was translated into a set of time-consuming management tasks done by hand:

(L1) The log files analysis to define the students expertise. Teachers counted from the log files the tags visited per student. Each student was defined as an expert in a particular building after the tag recount and the study of his/her preferences extracted from the questionnaire. Manually analyzing the log files was hard to carry out without errors and was very time demanding. To this, it was added the complexity of forming groups during the first weeks of the course: typically, some students drop out during this period and the groups had to be re-organized more than once. For all these reasons, the second phase of the activity was performed one week after the exploration.

(L2) Another detected limitation was the difficulties and limitations of using Moodle to assign the different activities to each of the groups and to show to each student the learning flow corresponding to this group. Moodle does not facilitate the automatic distribution of the appropriate activities among the different groups. The teachers employed the e-mail and the Moodle forums to inform each of the groups which of the activities to perform at each phase.

(L3) Finally, replicas of the course were very difficult to obtain, which forced selecting $2 \mathrm{~d}$ for performing the activity. The consequence was that not all the registered students could participate in the experiment (241 enrolled students, 74 actual participants).

\subsection{Requirements}

From the limitations of the script listed in Section 2.2, here we extract a list of requirements that the new version of the script should accomplish. Table 1 summarizes the relationship among the specific limitations and the requirements.

(Req1) Reusability: the solution should support script reuse with a reasonable cost for the teacher and in a short time period.

(Req2) Adaptability: the solution should support the selective delivery of content material, depending on each learner's characteristics. It should also facilitate the teacher in organizing the students in groups and distributing the tasks among them.

(Req3) Balance of guidance and flexibility: the script should guide the participants across its different phases, while the orchestration system should support the teacher in the management of unexpected situations.

(Req4) Scalability: the solution should be able to be used with a large number of students.

From the list of requirements, reusability (Req1) and scalability (Req4) are requirements that appear in many educational technology research projects. On the one hand, activities that are technologically supported need to be easily replicated with a reasonable cost for practitioners. On the other hand, these technologically supported activities have to be deployed in contexts with a large number of students. The accomplishment of these requirements might facilitate the adoption of these technological systems in actual educational contexts.

However, adaptability (Req2) and guidance and flexibility (Req3) are technological requirements directly related to the specific characteristics of CSCBL scenarios. A technological support 
Table 1

Summary table of the relationships among limitations and requirements.

\begin{tabular}{ll}
\hline Limitations & Requirements \\
\hline $\begin{array}{l}\text { (L1) Time consuming group } \\
\text { management }\end{array}$ & (Req4) Scalability \\
(L2) Lack of integrated method to guide & (Req3) Balance between guidance \\
students & and flexibility \\
(L3) Difficulties in reusing the course & (Req2) Adaptability \\
& (Req1) Reusability
\end{tabular}

for CSCBL has to provide the teachers with the necessary tools and mechanisms to organize the student groups and to distribute these tasks among the groups. At the same time, the system should facilitate the teacher to adapt the activity flow of the different groups and students involved in the activity according to their actions in their previous activities. For example, in the CSCBL scenario analyzed for this study the system should provide the students with the guidance necessary to help them to continue the activity. Also, the system should be flexible enough to support the teacher in facing "last minute changes" that may be required to modify the groups and task distribution.

There exists some solutions for the enactment of learning scripts, many of them based on the use of IMS LD. However, to the knowledge of the authors of this paper, none of them allows the integration of blended elements while guaranteeing the above mentioned requirements. A comprehensive revision of the state of the art can be found in [22].

\section{An architecture for the support of the CSCBL script}

In this paper we propose an orchestration system that combines various technologies to meet the requirements listed in Section 2.3 and overcome the limitations detected in the previous edition of the script as discussed in Section 2.2. This orchestration system supports both macro and micro scripts [11], which are the two levels of a CSCL script. This fact sets the basis of an underlying architecture to support generic CSCBL scripts. For the sake of clarity, in this section we present the orchestration system, the formalization process of the activity flow with IMS LD and how this activity flow is enacted. Finally, we describe the underlying software architecture and the design process followed to design the CSCBL script.

\subsection{The orchestration system}

The orchestration systems is based on the integration of several tools to support the needs identified in the activity flow. Depending on the supported tasks, we distinguish between those technologies to support the activities (learning activities support) and those to facilitate management tasks (orchestration support). All these technologies are integrated so that the course participants (both teachers and students) can focus on learning activities while reducing to a minimum the impact of management tasks. The following subsections describe these technologies and how they are integrated.

\subsubsection{Technological support for the learning activities}

- Mobile phones with Near Field Communication (NFC) Player. For the "Discovering the campus (2010 Edition)" phase students were given a mobile phone model NOKIA N6132 or N6212. These devices have an embedded NFC Player. NFC is a wireless technology allowing the exchange of data between devices located closer than $10 \mathrm{~cm}$. The devices had the application NFC Player [23] pre-installed. This application identifies an RFID card close to the device and reproduces a multimedia content previously associated with such card. This touch to learn interface allows the creation of interactive physical scenarios with cards in various spatial locations.
- gMapsQTItest. This tool is an implementation of the QTIMaps model [24] that combines the IMS QTI standard with web maps services for enabling the computational assessment of geographical skills [24]. gMapsQTItest uses Google Maps as the web map service, provides an editor for creating the tests, and a runtime system to detect and record the answers when users interact with the maps.

The mobile phones and the NFC application are used to access the information attached to the RFID tags distributed along the campus. gMapsQTItest is used by the students in the last phase "Reflect about the campus". They answer the questionnaire about various campus buildings interacting with Google Maps, and the system automatically corrects the answers.

\subsubsection{Technological support for the orchestration}

- IMS Learning Design. This specification released in 2003 [25] is used to formalize a sequence of activities, called a Unit of Learning (UoL), that can be deployed and enacted several times in a compliant platform such as GRAIL [26]. Section 3.2 details how the learning flow of the experience has been formalized with IMS LD.

- Google Spreadsheets. Google Docs is a web based office suite that offers the most common functionality for word processing, slide presentations and spreadsheet based data management. The spreadsheet functionality includes Google Forms, a tool to create and publish web forms so that the answers are automatically stored in a spreadsheet. The proposed orchestration system uses Google Spreadsheets and Forms to facilitate the creation of surveys and to simplify management tasks derived from the collected data.

- Log processing. The NFC Player installed in the mobile phones records a set of events in the form of log files. These files are parsed and processed to produce a summary of their content in $c s v$ format for further analysis.

- Generic Service Integration (GSI). This is a pluggable architecture that allows automatic configuration and bidirectional information exchange between IMS LD and external web based tools such as Google Docs [27]. GSI achieves this integration by a mediation that translates IMS LD requests into queries understandable by various tool-specific APIs. This mediation requires the implementation of an adapter and its configuration.

The GSI service adapter presented in [27] allows the integration of Google Forms and Spreadsheets in the context of a UoL: the questionnaire is presented to the students as a regular activity, and the data collected by the form and stored in the spreadsheet can be retrieved by the IMS LD player and used for orchestration purposes.

The IMS LD specification is recognized by many authors as the de facto standard to capture the sequence of activities in a learning experience [28-32]. IMS LD supports the formalization of a wide range of pedagogical models, including collaborative and adaptive learning flows $[33,34]$. The course flow of the experience described in this document was formalized as a UoL so that all the activities are orchestrated and managed by an IMS LD player.

Despite the effort to raise the adoption of the IMS LD specification in real life learning scenarios, the reality is that it has not reached the expected levels of adoption by educational institutions. The specification presents several advantages and innovations when compared to other systems, but the original design also present some drawbacks that limit its usage. For example, [35] discusses various drawbacks such as the manifestcentered course life cycle, the complexity of level B notation, or the lack of usability offered by current tools. The absence of a mechanism to handle the flow of artifacts is pointed out by [36]; while the support provided for collaborative settings is criticized 


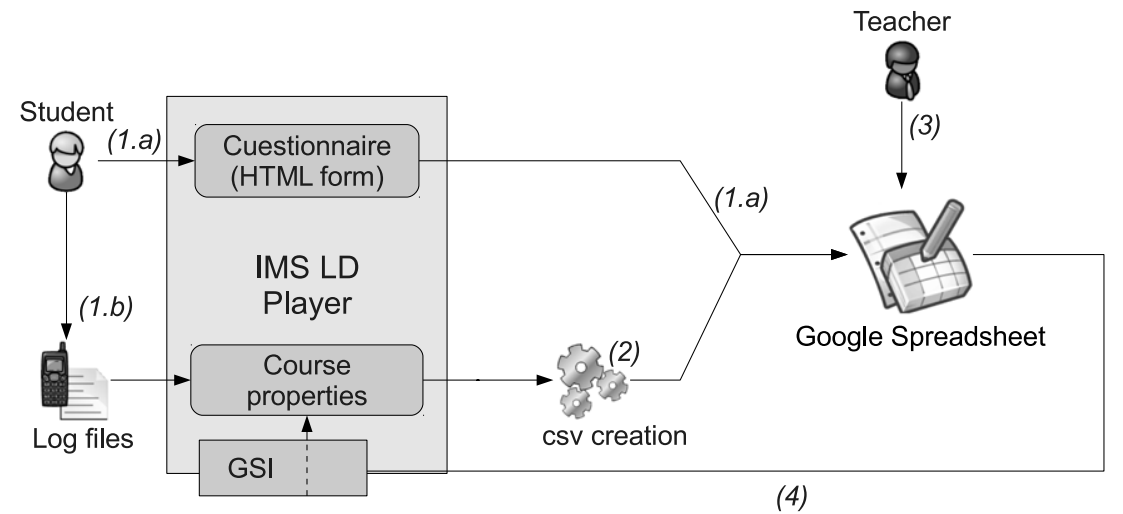

Fig. 1. Data flow in the orchestration system.

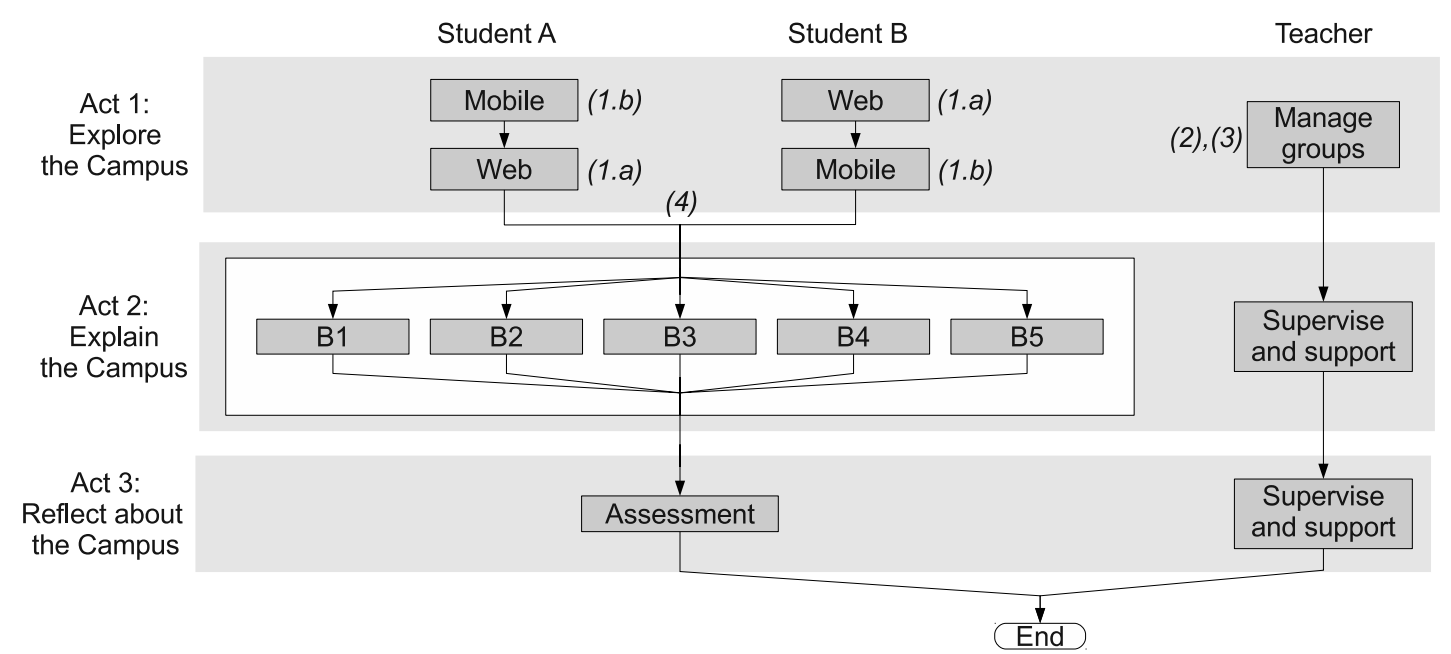

Fig. 2. Formalization of the activity flow with IMS LD.

by [37]. Other contributions [38,39] propose extensions to enhance the support for adaptive learning or the integration with intelligent tutoring systems.

In spite of all these drawbacks, several cases of study have demonstrated the suitability of the IMS LD specification for the deployment of complex activity flows. For example, [40] explored the use of Collaborative Answer Negotiation Activities with IMS LD, and [41] used the specification to orchestrate Problem Based Learning methodologies. Apart from its sequencing capabilities, IMS LD also facilitates the reusability of learning courses. The GSI extension was proposed to improve IMS LD by allowing generic tools to be integrated in a conventional activity flow [27]. The work described in this document uses IMS LD reusability to comply with requirement Req2, and evaluates how GSI can overcome the IMS LD drawbacks when used in CSCBL scripts.

The enactment of the resulting UoL can be summarized as follows (see Figs. 1 and 2). First, the course participants log into the player and access the description of their assigned activity. After their participation, the system contains two additional resources: the log files captured with the mobile phones (labeled in Fig. 1 as 1.a) and the answers to the Google Forms questionnaire (1.b).

The log processor processes the log files generated during the exploration phase and produces a $\operatorname{cs} v$ file with the events generated by each student (2). This file contains, for each student, the number of tags accessed per building and the building expertise, which is the building with the maximum number of accessed tags. Such information is then automatically incorporated to a spreadsheet containing the students' responses to the Google Forms questionnaire. Then, Google Spreadsheets is used for the group management: the teacher reviews and manipulates the collected information and creates the student groups (3).

Finally, the GSI framework triggers a data synchronization step between the IMS LD player and the Google Spreadsheet (4), so that the group information in the spreadsheet is transferred to the IMS LD player for further orchestration.

\subsection{Formalization of the flow with IMS $L D$}

The formalization of the learning flow with IMS LD involves two main aspects: first, the definition of the activity flow in terms of the IMS LD vocabulary; second, the use of GSI tools to establish how and when the information is exchanged between the IMS LD server and the spreadsheet. That is, the learning script must contain a description of the case specific tools (i.e. Google Spreadsheets), with the required vocabulary as defined by GSI [42].

As depicted in Fig. 2, the IMS LD flow is composed by three acts corresponding to the three phases of the course. Three roles (two students and one teacher) are used to classify the course participants, where the difference between the two student roles is the order in which they follow the activities in the first phase: students of type A start with the web exploration; students of type B start with the exploration using mobile phones. Each student decides when the first activity finishes to continue with the second one. That is, the completion condition is user-choice. The first act is completed when the teacher completes his/her role-part.

In the second act, explain the campus, each student access one of the five available activity descriptions. Which activity is accessed is determined by the corresponding conditions imposed to the 


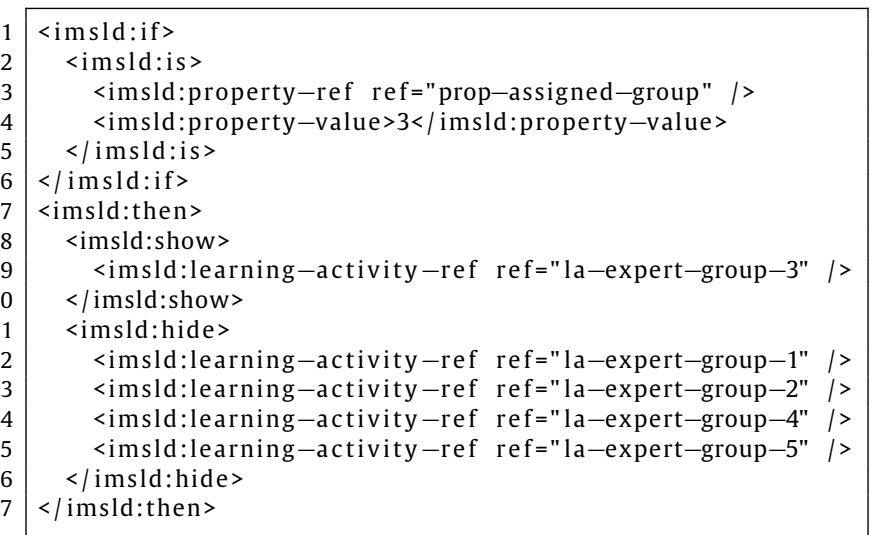

Listing 1: "Conditions used in the IMS LD manifest to show and hide content".

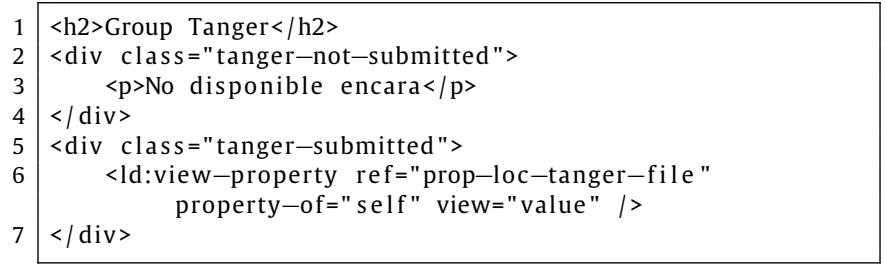

Listing 2: "Use of the view-property element".

group_number property. That is, the value of such property guides the student to the appropriate activity, as shown in Listing 1, depending on his/her actions during the exploration phase. The name of all group members is stored in another property, which is shown to the students in order to inform who their team mates are. The activity in this second act finishes when students submit a document which is stored as the value of the corresponding rolescoped property. As a consequence, all the submissions are stored with a regular structure and can be easily reused in the last act. The second act finishes when the teacher completes his/her role-part.

In the third act, students access the QTI + Google Maps so that they complete the final assessment. To foster a reflection process, they can access the documents submitted by other groups. This information flow is modeled in the UoL as an imsldcontent document with the properties created in the previous phase containing the group documents created in the previous act (see Listing 2).

Modeling teacher tasks is simpler than in the case of the students: each act contains a single activity with a description of what the students are doing and what the teacher can do to support them. The completion condition for all teacher activities is userchoice and their completion causes the corresponding act to be set as finished. Fig. 3 shows the user interface of the IMS LD player used to reproduce the described sequence. It is important to note that the interface shows the description, but the activity is carried out in an external tool.

The information exchange between the spreadsheet and the IMS LD player is triggered by the teacher activity: when all the data is stored in the spreadsheet, the teacher sets his/her first act as finished. As a response to this event, GSI synchronizes the data between the IMS LD player and the spreadsheet, filling all the values of the student property group_number. Listing 3 shows the XML description of the action that triggers the data retrieval at the IMS LD player. Once all students have been assigned to a group, the system delivers the activity corresponding to the second act.

The script used in the case of study was based on the activity flow described in [17] modified with various enhancements

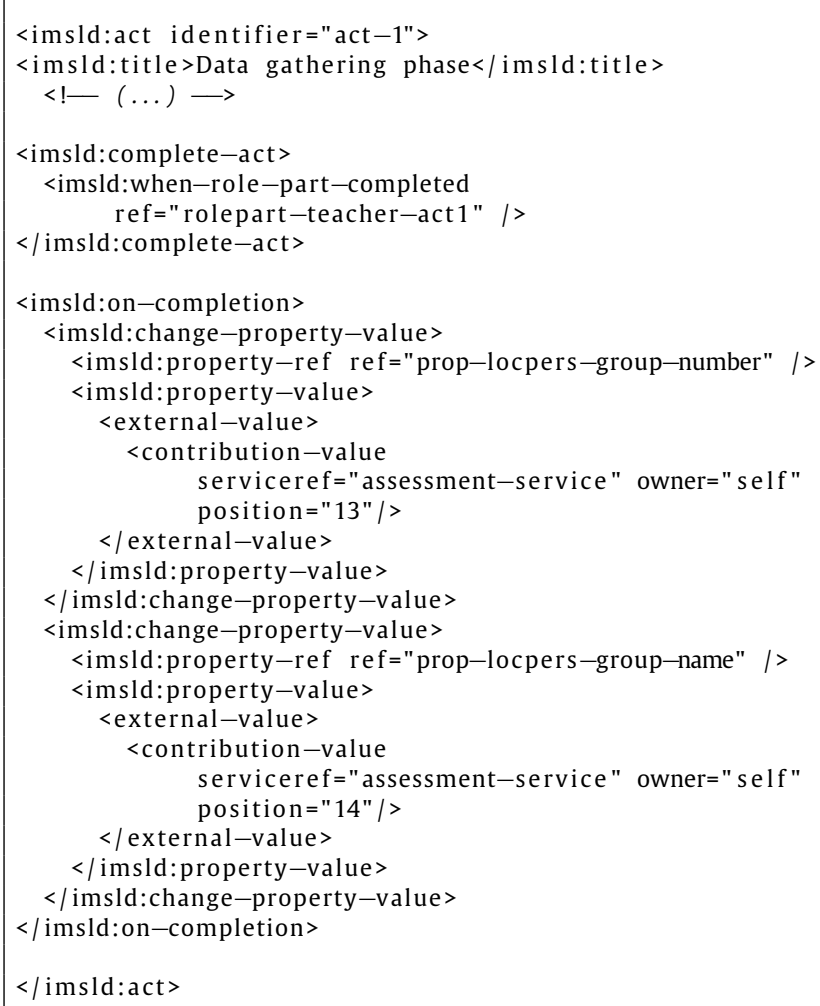

Listing 3: "Trigger for data retrieval at the IMS LD player".

suggested by the teaching staff. Due to the lack of authoring tools supporting GSI features, the script was created by the researchers. A pattern-based approach to authoring as the one presented in [33] would allow this step to be carried out by the teaching staff instead. However, authoring issues were set aside to favor the assessment of the orchestration capabilities of the system.

\subsection{The enacted activity flow: 2010 edition}

Section 3.2 showed how the proposed learning experience was expressed using IMS LD. In this section the experience is described from the point of view of the students. The experience was structured as a sequence of activities presented to students as three phases:

- "Explore the campus": Students gathered in the classroom and used their university account to access the IMS LD player and start the experience. This first phase consisted of two exercises: a web exploration and a mobile exploration. Half of the students started with the web exploration and continued with the mobile exploration. The other half did the other way around (see a description of the activity flow in Section 2). In the web exploration exercise, students were asked to explore the web page describing the campus services and answer some questions. In the mobile exploration exercise, students were given a mobile device and were asked to interact with the NFC tags distributed around the campus to learn about the different areas. After the exploration with the mobile phones, the students had to come back to the classroom and return the mobile phones to extract the log files using a Bluetooth interface.

- "Explain the campus": This phase consisted in preparing a document explaining the main characteristics and services of one area of the campus working in groups. Each student was redirected to an activity group that indicated who their 


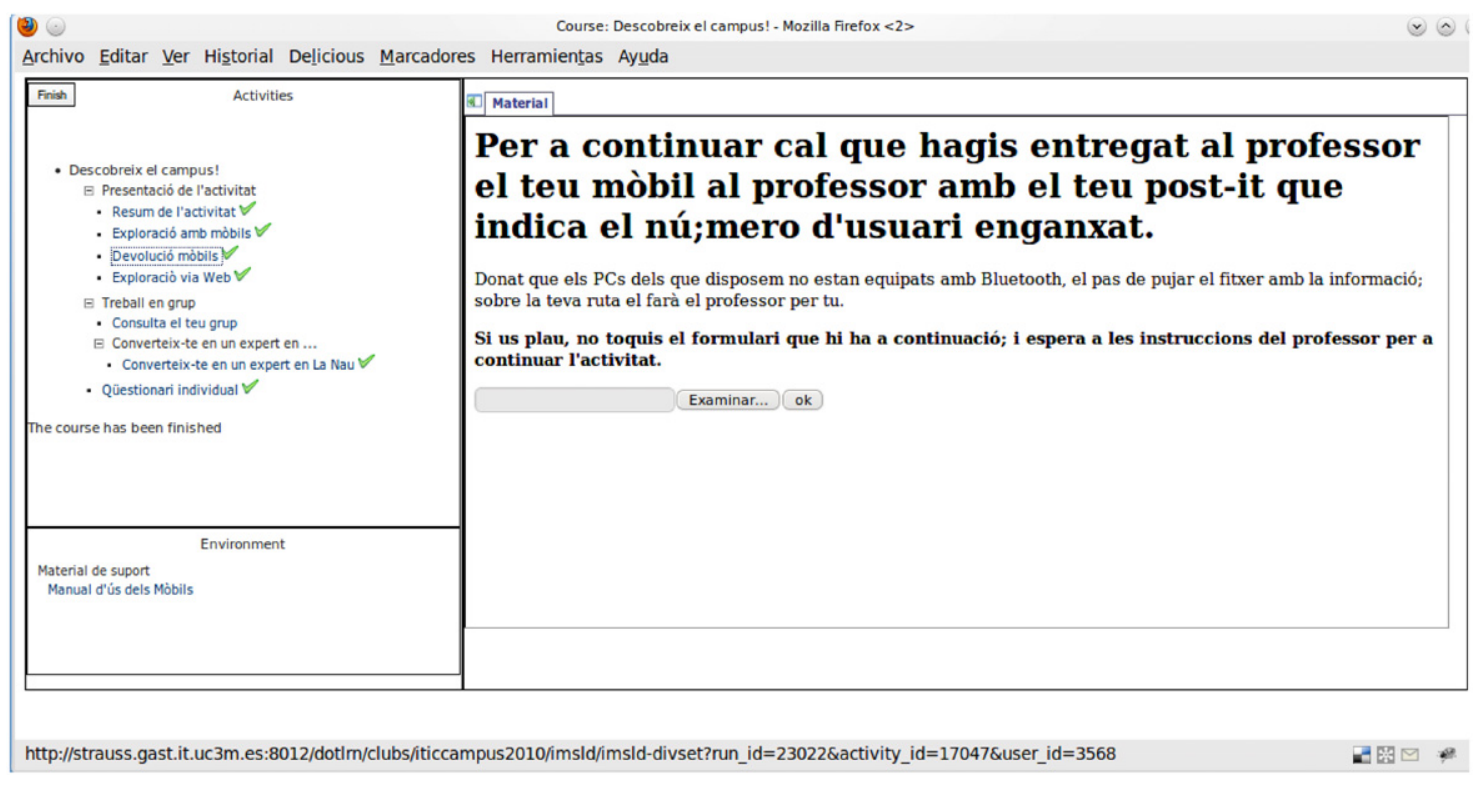

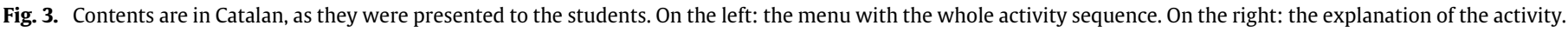

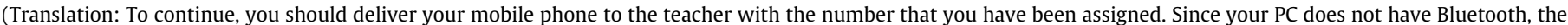

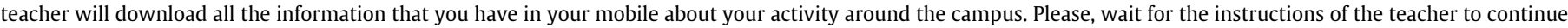
the activity.)

team mates were and the area of the campus assigned. All the students had to first find their team mates and then sit together in front of the same computer. The group collaborated to prepare the final document about the assigned campus area, which served as the input data for the final phase. Once finished, the group had to mark this activity as completed.

- "Reflect about the campus": In this phase the students accessed a questionnaire about the campus. This questionnaire contained a set of questions to be answered by interacting with a Google Map of the Campus. Students answered questions by selecting one of the various options located in the map. To answer the questionnaire, students could access all the documents generated by the rest of their colleagues in the previous phase. With this approach, all the students could learn from their colleagues about the different campus areas. Once the questionnaire was solved, the students received feedback about their marks according to their performance.

Two adjustments of the learning flow with respect to the first version of the experience were required. First, only the teacher computer was equipped with a Bluetooth interface so that the students were not able to upload the log files generated by the mobile phones. This task was performed by the teacher, who uploaded the log to the computer and used GRAIL's administration facilities to set the student's property value with the corresponding $\log$ file. The other modification is related to the group formation process: the teachers were not familiar with the script used to parse the log files and no user-friendly interface was provided. Due to this fact, the group formation process was performed by the researchers under the supervision of the teacher. These two adjustments neither affected the enactment of the script nor did they bias the resolution of the research question.

\subsection{The underlying architecture}

Section 2 presented the limitations found in a case of study that was devoted to the deployment of a CSCBL script. Then, after the identification of the requirements for an effective support of such script, this section has proposed an orchestration system that integrates, as a solution for the limitations found in a specific situation, several tools for the support of the learning and management activities. From the proposed orchestration system we now derive an underlying architecture that can be reused for the support of CSCL and CSCBL scripts different from the one presented in this paper.

According to [11], two levels can be identified in a learning script. First, the macro script level, which stands for the overall sequence of activities that produces the desired interactions. Second, the micro script, which refers to the scaffolding of the interaction per se. The distinction between micro and macro is not binary and depends on the specific details of the script, but both of them require to be supported by the orchestration system.

While existing frameworks for the use of learning scripts focus on supporting macro scripts (e.g. IMS LD claims to allow the use of a wide range of pedagogical models), they provide neither the functionality nor the flexibility expected for the scaffolding of micro scripts, where case specific tools would better support the task. Furthermore, the activities of the micro script level may happen at different spatial locations, thus hindering a centralized management of the flow.

Despite requiring support from different tools and happening at different spatial locations, there is a strong relationship between these two levels and one require the input from the other. GSI scaffolds the needed relationship between the two levels and, thus allows the orchestration system to support both macro and micro scripts. The bidirectional exchange of information among tools allows the automatic configuration of the case specific tool depending on the state of the macro script as well as the adaptation of the activity sequence depending on the interactions that take place in micro script.

The architecture that underlines the technological solution presented in previous Section 3.1 is based on the use of IMS Learning Design to define and deliver the main activity sequence (i.e. macro script) and case specific tools (NFC mobile phones, Google Spreadsheets, log processing) for the scaffolding of collaborative interactions across spatial locations (micro scripts).

The architecture proposed is summarized in Fig. 5. At the macro script level, that is, within the IMS LD run time environment, the course participants access the player to obtain the instructions for their next activity. They are then enabled to perform a number of 


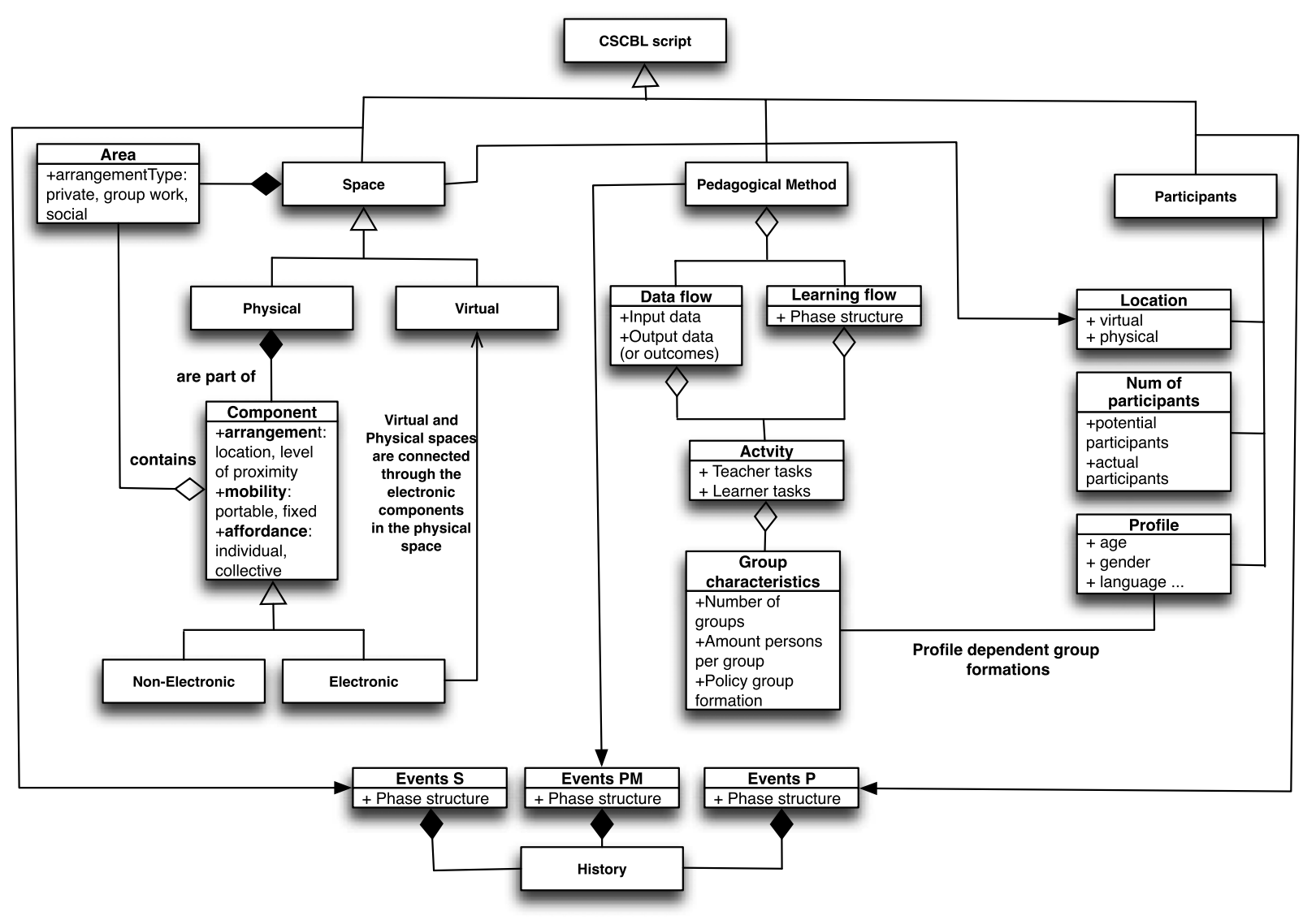

Fig. 4. 4SPPIces Model. Factors and facets to be considered in the design of CSCBL scripts and of the technological environment for supporting their enactment.

actions such as access the content, mark activity as finished or submit a solution. These actions may trigger the conditions imposed to the collaborative learning flow, and therefore they may change the state of the script. For example, if a student submits a document in a certain activity, her team mates will find that the corresponding activity has been automatically marked as finished.

GSI acts as a communication layer and enables such triggers to exchange information with third party tools, which provide the case specific scaffolding needed at the micro script level. In such a case, there are two possible interaction types: first, the students access the third party tool via the user interface provided by the tool. Second, the IMS LD player exchanges information with the tool in the background. From the point of view of the IMS LD player, the case specific tool is a black box that informs when a certain action has been executed. From the point of view of the students, the case specific tool provides the functionality required to properly execute the proposed activity, which may happen at different spatial locations. The proposed architecture could be used in other learning situations by simply substituting the case specific tools for those that better fits the case or the spatial situation.

In summary, the proposed architecture is based on three key elements: first, macro script support provided by a flow management framework; second, case-specific tools for the support of micro script interactions; finally the connection of these two levels via GSI, a communication layer that allows bidirectional information exchange among tools during the enactment of the script. The presented orchestration system might therefore be useful for any CSCL script that require these three elements to be supported.

\subsection{CSCBL script design process}

Both editions of the experience, the 2009 and 2010 edition were designed according to the 4SPPIces model proposed by the authors of the paper in a previous study. 4SPPIces is a conceptual model that provides practitioners and technicians with a common language to design CSCBL scripts and the technological setting for supporting their enactment [12]. 4SPPIces defines 4 factors: (1) the Space, which defines the planned environment where learning activities are going to take place, (2) the Pedagogical method, that defines a learning flow, (3) the Participants, which defines the people involved in the activity and their characteristics and (4) the History, which models those aspects from the other factors likely to be affected by the unpredictable variations usually produced during the scripts enactment 4.

In the 2009 edition, the CSBL script was designed as follows. The Pedagogical Method factor was divided into three phases: (1) an exploration of the campus in which students access with mobile phones to the information hidden on 46 RFID tags distributed around the campus, (2) an activity in expert groups to prepare a presentation about a campus area and (3) an individual questionnaire in Moodle. The Participants' factor modeled the profile of the students, defined by their expertise on a campus area. The Space factor was the campus areas, the classroom and home. The History modeled the log-files that collected the actions of the participants around the campus to define the students' expertise and form the groups for the following phases accordingly.

In the CSCBL script for the 2010 edition, we maintained the original design from the 2009 edition but we varied the architecture implemented for supporting its enactment to provide complete automatic support of the script. The main difference between the two scripts is the technological support employed for representing the Pedagogic Method factor. In the first case we used Moodle while, in the second one, we selected IMS Learning Design for codifying the Pedagogical Method factor. Also, we used the Generic Service Integration (GSI) system during the enactment, to manage students 


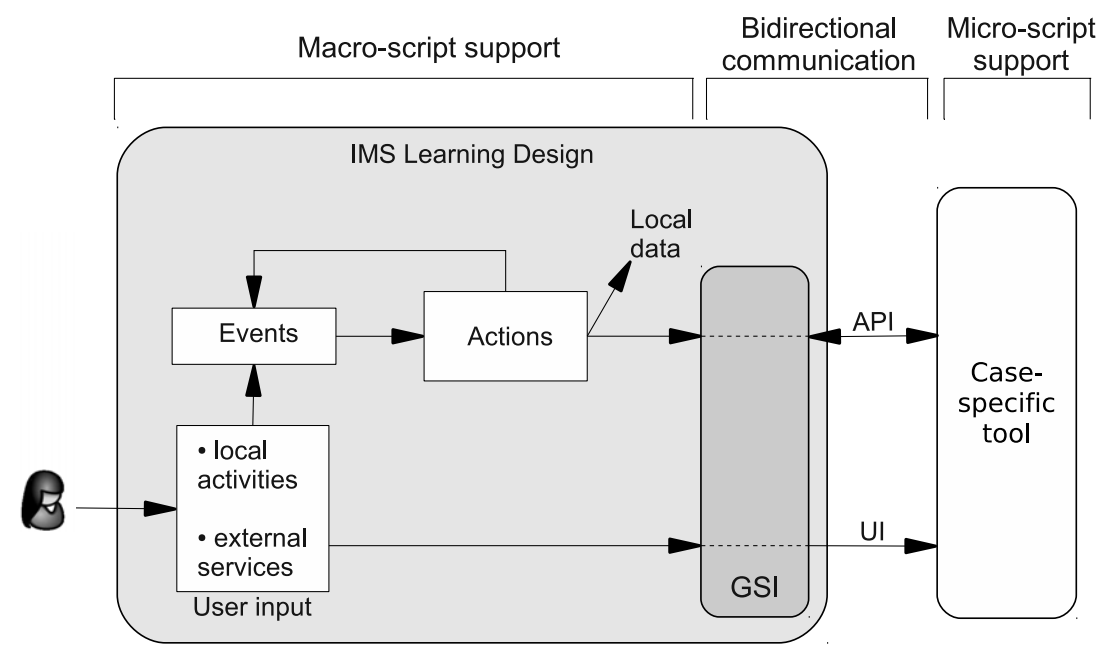

Fig. 5. Underlying architecture of the proposed orchestration system.

data (e.g., mobile phone log files) and automatically create groups by manipulating a Google on-line spreadsheet integrated with the LMS.

Both CSCBL scripts designs are generalizable for any other learning context with similar educational objectives. Since the two scripts were designed according to the 4SPPIces factors they share commonalities in their design but vary in the technology employed to support their enactment. Any other researcher or practitioner could adapt these CSCBL scripts according to the 4SPPIces model and re-configure some design aspects according to his/her learning interests. Then, they could reuse the architecture proposed in this paper or implement a new one that better fits with their interests.

\section{Evaluation}

Although generalizable to other situations, the presented orchestration system comes from a very specific requirement: to overcome the limitations found in a previous learning experience. It is then reasonable to focus the empirical evaluation towards determining whether or not the limitations have been effectively overcome. However, solving the limitations of the previous experience is not sufficient. The orchestration system can be considered an improvement of the previous solution if it maintains the already observed learning benefits.

It is the authors statement that the orchestration system improves the previous script, and therefore the empirical evaluation must determine (1) if the system provides a solution for the detected limitations and (2) if the script is pedagogically equivalent to the previous one. These two different requirements will be covered in different focuses, as presented in Section 4.2.1.

This section first describes the case study. Second, it explains the data analysis methodology; finally, it presents the main results of the analysis.

\subsection{Description of the case of study}

The CSCBL script was included as an activity of the course Introduction to ICT with first-year engineering students of the University Pompeu Fabra. It was offered to the students as an optional activity, and they received no extra reward for their participation. For administrative purposes, the students were requested to register in advance and to apply for a specific course instance among 4 offered options. Each of these instances was tutored by a different person. That makes 4 voluntarily involved teachers, two of which were the coordinators of the Introduction
Table 2

Number of participants in the different course instances.

\begin{tabular}{llc}
\hline Instance number & Timetable & Number of participants \\
\hline 1 & $10: 00-12: 00$ & 9 \\
2 & $12: 00-14: 00$ & 17 \\
3 & $14: 00-16: 00$ & 3 \\
4 & $18: 00-20: 00$ & 3 \\
\hline
\end{tabular}

to ICT course, and the other two were invited secondary school teachers familiar with ICT applied to education. The enactment of each instance was independent from the others and, apart from the timetable, there were no difference among them. That is, they had the same initial configuration, but different participants. The script was designed to support 25 students per instance. In the authentic setting, a total of 32 students participated in the experiment (see Table 2). The researchers participated as observers and technical supporters.

The replication method for each of the instances was the same and consisted in the following actions:

- The teachers, who were already familiar with activity sequence, were trained on how to use GRAIL. Such training took place 15 min before the students arrival.

- The students, once they arrived, were introduced in GRAIL as the interface through which they would receive activity descriptions. That is, students were not told what to do, but they received instructions on how to know what to do.

- Once both teachers and students had been introduced to the environment, students were numbered so that they received a unique username and password to login in the system.

- Finally, a course instance, in the sense of IMS LD, was populated with the participants and then instantiated.

\subsection{Data analysis methodology}

The evaluation of the experiment considered different data sources (detailed in Section 4.2.2) to find an answer for the research questions (stated in Section 4.2.1). According to the analysis method explained below, there is no a priori explicit correspondence among data sources and research questions. The results discussion in Section 4.3 establishes the a posteriori correspondence by relating the findings with their supportive data (Table 2 and 7).

The data extracted from the four course instances was analyzed following a mixed method evaluation [43]. That is, data was gathered from different perspectives, each of them offering a different view. Mixed methods combine quantitative techniques, 
Table 3

Focus 1: summary table of the findings regarding the technological focus.

\begin{tabular}{|c|c|}
\hline Findings focus 1 & Supportive data \\
\hline $\begin{array}{l}\text { 1.I. The CSCBL script have been successfully reused } 4 \text { times, with a reasonable cost that allowed the enactment of the four two-hour } \\
\text { replicas in a total of } 8 \mathrm{~h} \text {. }\end{array}$ & $\begin{array}{l}\text { [Observations] } \\
{[\text { Interview] }} \\
\text { [TeachersQuest] } \\
\text { [StudentsQuest] }\end{array}$ \\
\hline $\begin{array}{l}\text { 1.II. The orchestration system successfully adapted the learning material to the individual circumstances of the students and group } \\
\text { membership, considering the information stored in distributed tools. }\end{array}$ & $\begin{array}{l}\text { [Interview] } \\
\text { [TeachersQuest] } \\
\text { [Observations] }\end{array}$ \\
\hline $\begin{array}{l}\text { 1.III. The CSCBL script supported and facilitated students in following the activity, and offered a flexible enough system that supported the } \\
\text { management of the particular unexpected situations that arose during the experiment (see Table } 6 \text { ). }\end{array}$ & $\begin{array}{l}\text { [Interview] } \\
\text { [StudentsQuest] } \\
\text { [Observations] }\end{array}$ \\
\hline
\end{tabular}

Table 4

Summary table of the numeric answers given to the questionnaires.

\begin{tabular}{|c|c|c|c|c|c|}
\hline & Question & Legend & Mean & $\begin{array}{l}\text { Standard } \\
\text { deviation }\end{array}$ & $\begin{array}{l}\text { Confidence interval for } \\
\text { the mean }\end{array}$ \\
\hline 01 & $\begin{array}{l}\text { Does the time between activities breaks the } \\
\text { overall pace of the flow? }\end{array}$ & $\begin{array}{l}1=\text { no, it does not break the pace } \\
5=\text { yes, it breaks the pace }\end{array}$ & 2.05 & 1.07 & {$[1,2.45]$} \\
\hline 10.a & $\begin{array}{l}\text { Assess how difficult was to identify the activity } \\
\text { you had to do }\end{array}$ & $\begin{array}{l}1=\text { very easy } \\
5=\text { very difficult }\end{array}$ & 2.35 & 1.28 & {$[1,2.74]$} \\
\hline 10.b & $\begin{array}{l}\text { Assess how difficult was to go to the next activity } \\
\text { once you have finished }\end{array}$ & $\begin{array}{l}1=\text { very easy } \\
5=\text { very difficult }\end{array}$ & 2.32 & 1.4 & {$[1,2.67]$} \\
\hline $10 . c$ & $\begin{array}{l}\text { Assess how difficult was to understand } \\
\text { what to do }\end{array}$ & $\begin{array}{l}1=\text { very easy } \\
5=\text { very difficult Yes }\end{array}$ & 2.29 & 1.29 & {$[1,2.68]$} \\
\hline 11 & $\begin{array}{l}\text { Have you need the help of the teacher } \\
\text { at any moment }\end{array}$ & $\begin{array}{l}\text { No } \\
1=\text { bad integration } 5\end{array}$ & $\begin{array}{l}15 \text { yes } \\
16 \text { no }\end{array}$ & - & - \\
\hline 12 & $\begin{array}{l}\text { Assess the perceived integration of the different } \\
\text { technologies and activities }\end{array}$ & $\begin{array}{l}=\text { good integration } \\
\text { Yes }\end{array}$ & 3.84 & 0.68 & {$[3.63,5]$} \\
\hline 18 & $\begin{array}{l}\text { Would you recommend this course } \\
\text { to your friends? }\end{array}$ & $\begin{array}{l}\text { No } \\
\text { NA }\end{array}$ & $\begin{array}{l}29 \text { yes } \\
1 \text { no } \\
1 \text { NA }\end{array}$ & - & - \\
\hline
\end{tabular}

such as closed questions, with qualitative techniques such as open questions, discussion groups or observations. Due to the inherent subjectivity on the qualitative analysis and, in order to increase the validity of the findings, we used a method called triangulation. Triangulation consists in reinforcing each of the interpretations extracted through a comparative analysis of the evidence provided from different sources. That is, to analyze each conclusion from a different perspective in order to have several confirmations supported by both qualitative and quantitative data. The concrete data sources are presented in Section 4.2.2. This paper uses fragments of the extracted data, while the whole raw data and the tables with the partial results obtained during the process can be accessed at http://mozart.gast.it.uc3m.es/ mmpsanag/ escolab2010/.

In order to ensure its validity and significance, the qualitative data was statistically analyzed by applying the $t$-test. Thus, numeric data is presented by its mean, 95\% confidence interval for the mean, and standard deviation. For the sake of readability, Table 4 presents these values, for each analyzed question.

\subsubsection{Research focuses}

The presented case study is based on a previous script where some limitations were detected (Section 2.2). A critical analysis of such limitations resulted in the statement of specific requirements that drove the development of an orchestration system (Section 2.3). Therefore, the validation of the orchestration system is twofold: first, the empirical validation of the technical capabilities of the orchestration system in relation to the requirements; second, the empirical analysis of the computer supported scripts in comparison with the previous, non computer supported, version of the script.

The first analyzed focus relates to the capability of the technology to effectively support the orchestration and execution of the planned activities, and how the requirements imposed by the CSCBL script (see Section 2.3) are satisfied. In other words, how good was the system formed by IMS LD and GSI on the activity orchestration and what difficulties were found. The specific research questions are:

(RQ1) Is it feasible to reuse the CSCBL script, with different participants, several times with a reasonable cost and with independence among the different instances? This question relates to Req1.

(RQ2) Does the orchestration system provide a means to capture and process the students information so that the delivered content can be adapted to their individual characteristics and group membership? This question relates to Req2.

(RQ3) Does the orchestration system provide guidance to teachers and students, at the time that offers enough flexibility to support unexpected situations management during the enactment? This question relates to Req3.

(RQ4) Can the orchestration system be applied to a large number of participants with a reasonable cost? This question relates to Req4.

The second focus is related to the educational value of the CSCBL script. In other words, whether the CSCBL script helps on the acquisition of the expected learning outcomes and if it is equivalent in terms of learning benefits with the first edition of the activity. The specific research questions are:

(RQ5) Does the mixture of formal and informal activities integrated into the CSCBL script support students' reflection about the individual campus exploration with mobiles?

(RQ6) Does the CSCBL script enacted support knowledge acquisition within the campus?

(RQ7) Does the CSCBL script enacted help students to meet people?

(RQ8) Does the CSCBL script enacted motivate students? 
In summary, the first focus is oriented towards an empirical evaluation of the proposed orchestration system: a previous study led us to the definition of a technological solution for the encountered limitations, and the answer for research questions 1-4 will tell us to what extent those limitations were overcame. The second focus attempts to analyze the pedagogical issues of the computer-supported situation. The CSCBL field is relatively new (specially regarding the 'blended' part) and there is still a need the characterization of such type of scripts. That is, the second focus is oriented towards the qualitative exploration of the scenario.

\subsubsection{Data sources}

According to the used mixed method evaluation technique, different data sources were evaluated on the analysis of the experiment:

TeachersQuest After all activities of the course, an anonymous survey was provided to teachers. The data gathered was both qualitative (e.g. open questions) and quantitative (e.g. closed questions and Likert-scaled questions).

StudentsQuest After all activities of the course, an anonymous survey was provided to students. The data gathered was both qualitative (e.g. open questions) and quantitative (e.g. closed questions and Likert-scaled questions).

Observations The four course instances provided valuable information that was captured during their instantiation and enactment. 5 external observers, i.e. persons who neither participated nor interfered in the activities, made annotations on the facts that considered noticeable.

Interview One of the teachers, an actual member of the teaching staff of the ICT introduction course, was interviewed after the finalization of the activity flow. She is a user with low technological skills that has only used basic Moodle functionalities in her courses and she was not familiar with the research intentions of the system. The type of interview was methodologically between an informal conversation and an interview guide approach [44]. The interview was recorded and transcribed for its later analysis.

\subsection{Results}

To increase the readability of the results, the findings of this experiment have been clustered according to the evaluation focuses explained in Section 4.2.1.

\subsubsection{Focus 1: technological support for the CSCBL script orchestra- tion}

Focus 1 is devoted to analyze whether the proposed technological system successfully supports the enactment of the CSCBL script, and accomplish all the orchestration requirements imposed by the learning flow.

The first finding under analysis (finding 1.I in Table 3) is related to the reusability of the CSCBL script (RQ1). The presented experiment hosted four different and independent instances of the learning flow supervised by different teachers. The reuse process allowed us to deploy and enact four instances of the two-hour script in a total of $8 \mathrm{~h}$, where course instantiation was done during the breaks between instances. From the absence of comments regarding the fact, it can be said that neither teachers nor students perceived any dependency with previous course instances. When questioned about perceived dependency among course instances, the interviewed teacher stated that "The course was self-contained. I did not perceive any connection" [Interview]. Furthermore, the teachers considered that the cost of the reuse process was not a limitation of the course, and that the instantiation process was understandable and not time consuming.

The answer to RQ2 (finding 1.II in Table 3) depends on the feasibility of the group formation process. That is, the students receive a version of the course material that will depend on the group to which they were assigned. As described in Section 3.3, the group formation depends on information generated in different sources and occurs once the students have started their activities, so that the required time may affect the performance of the overall course. Thus, the group formation process is said to be feasible if it can be performed in a reasonable time and does not break students' pace. Both teachers and students remarked that the elapsed time was not perceived as "something that breaks the overall flow". The interviewed teacher said that "the time waited for the group formation was reasonable" and pointed out that "even a longer time can be tolerated if you have a pre-programmed activity to fill this gap" [Interview]. On their side, the students considered that the time elapsed between activities was not perceived as a limitation for the overall pace: they averaged 2.05 where 1 meant "no, it does not break the pace" and 5 was "yes, it breaks the pace" (see Table 4) [StudentsQuest]. The results show that the use of a tool such as a spreadsheet, with which the teachers were said to be already familiar, in the group formation process was found useful and appropriate for hiding the complexity of the underlying adaptation scheme (i.e. IMS LD properties and conditions).

Another issue regarding adaptation is the integration of the used technologies in the complete orchestration system: a similar previous experiment [45] showed that the lack of integration among tools negatively affected the orchestration of adaptive learning content. The answers of question 12 from Table 4 show that the students perceived an integrated system. Also, the interviewed teacher recognized that she did not know that she was using tools from different providers. Furthermore, there are no reported problems while granting the IMS LD server with permissions to access Google data. However, the observations indicate that some of the tasks could be better integrated, such as the QTI questionnaire (with no integration at all) and the log analysis (where the parsing script consisted in a command-line based desktop tool) [Observations]. The view of the researchers is that the use of GSI for the integration of tools in IMS LD has improved the adaptation process within the orchestration system, and the participants perception are more positive than in previous experiences.

The third issue under discussion (RQ3) aims at analyzing the balances between the guidance through the learning activities and the flexibility of the orchestration system. Finding 1.III states that the course participants perceived an appropriate support for their activities. The analysis of the questionnaires shows that the students did not have remarkable problems following the activity flow. In other words, the orchestration system was able to support students' tasks. Answers from 10.a to 11 (see Table 4) reveal that the participants found the system easy to use. Table 5 shows the comparison of the perceived difficulty of the system among the students who needed teacher's help and those who did not. Despite the teachers perceiving a less usable system, the average of the answers reveals that their perception is positive. The questionnaires show that students satisfaction was high (question 18: 93.55\% would recommend the course to friends). The interviewed teacher recognized that she was able to know what to do at each activity, but she would have liked to had the chance to prepare the class in advance: "I did not feel constrained, the fact of having a step-by-step guide helped me on being self confident" [Interview]. The observations show that teachers understood the group formation process and found it helpful and appropriate to organize the students group; the interview and observations reveal that the teachers did not feel constrained by a rigid activity flow. 
Table 5

Comparative among students who asked the teacher and those who did not.

\begin{tabular}{llll}
\hline & $\begin{array}{l}\text { Asked the } \\
\text { teacher }\end{array}$ & $\begin{array}{l}\text { Did not ask } \\
\text { the teacher }\end{array}$ & $\begin{array}{l}\text { p-value (unpaired samples, } \\
\text { one-sided Wilcoxon test) }\end{array}$ \\
\hline $10 . \mathrm{a}$ & 2.87 & 1.87 & 0.01258 \\
$10 . \mathrm{b}$ & 2.67 & 2 & 0.03662 \\
$10 . \mathrm{c}$ & 2.8 & 1.81 & 0.01684 \\
\hline
\end{tabular}

The management of unexpected situations was also considered in the analysis of the provided support. Such situations appeared in all of the course instances and the teachers' reaction was possible with the use of the functionality provided by the orchestration system. Table 6 summarizes these situations and their solutions. The unexpected problems were mainly caused by students not respecting deadlines or dropping the course, and they were not an impediment of the course to be successfully enacted. Some of these problems are related to flexibility issues typically related to the enactment of scripted collaborative activities [15]. This is the case of the last three issues in Table 6, which have to do with aspects of group management. The system therefore offered a high level of flexibility that allowed overcoming unexpected situations that arose in the experiment. Moreover, the solutions proposed to deal with these problems could be easily adopted for the deployment of other CSCBL scripts or similar scripted collaborative activities.

Finally, RQ4 is devoted to the analysis of the scalability of the CSCBL script when enacted with the proposed orchestration system. That is, to analyze if the orchestration system is also applicable when the number of participants is large. Despite the presented experiment being designed to support 100 students, the number of actual participants was only 32 (probably because of the bad weather conditions). Such a number does not provide enough empirical evidence of a scalable orchestration system. However, the results reveal some scalability capabilities of the system. This claim is argued as follows: A collaborative pattern suits a certain number of participants (e.g. discussion groups should have less than 5 members in order to be effective), and it has no pedagogical sense to increase the number of participants. That is, the scalability of a CSCBL script refers to the feasibility of the course reuse process. In other words, a CSCBL script is scalable if it can be reused with a reasonable cost. The presented experiment was designed to support 25 students at each course instance but, in the actual setting, the maximum number of simultaneously supported students was 17 (see Table 2), during the second instance. Due to the lack of reported problems regarding the reuse process (finding
1.I) and the effective support provided to the students (finding 1.III), it can be argued that the orchestration system would have supported at least 17 students in the 4 course instances, so it was ready to provide support to more students than in the actual experiment.

Additionally, some limitations were detected in the orchestration system. First, the beta state of the supporting technology resulted in a usability problem to the teachers, who commented that the automatic parse and process was a little tricky and that they would have needed preparation to be comfortable with the system and its possibilities. This perception of trickiness was also caused by the user interface, which, it was said, was not user friendly enough. For example, one of the teacher comments: "(Teacher 1) I found it difficult to complete some of the tasks of which I was supposed to complete only for a lack of proper training on how to complete them. I feel that if I had a course (or several) before the actual session, training me how to perform the tasks depending on the specific situations, I would have been able to complete them without problem" [TeachersQuest]. The observations also show that the slowness of the server caused unexpected breaks during the activities (mainly during the group formation process) and that some students reported audio problems with the mobile phones. In summary, it can be said that the high number of tools in use required the attention of the researchers, which was not feasible in some cases.

Another reported limitation is related to the lack of integration of the QTI questionnaire: there was no means to automatically capture the score obtained by the students and set the corresponding IMS LD property value. This process had to be manually performed. Without knowledge of GSI, the teachers demanded an integration "similar to the spreadsheet integration". As a teacher commented in the interview: "I clearly saw the membership of the students in their building-teams, but when they had to answer the final questionnaire (with QTI) and fill a form with their marks, there it was not clear for me where to see such marks" [Interview]. In other words, the integration provided by GSI was positively perceived and demanded in all of the activities.

Finally, some students said that the exploratory activity was solitary and demanded a group activity such as a gymkhana. Some other students suggested the use of augmented reality instead of NFC interaction. These students comments are not really limitations of the activity flow, but suggestions to make it more interesting.

Table 6

Summary of unexpected situations and applied solutions.

\begin{tabular}{|c|c|c|}
\hline Issue & Solution & Tool \\
\hline Only one computer had a Bluetooth interface & The teacher uploaded all logs and updated students properties & Cockpit \\
\hline $\begin{array}{l}\text { Two students returned the mobile phone after the } \\
\text { deadline }\end{array}$ & They were manually assigned to a group, and their log data were ignored & Spreadsheet \\
\hline One student dropped the course & The user was ignored in the forthcoming activities & No action needed \\
\hline Only 3 students were in the course & The group formation was manually performed, with the 3 students in the same group & Spreadsheet \\
\hline Only one experts' group in the collaborative phase & $\begin{array}{l}\text { Documents from previous course instances was used as faked groups in the reflection } \\
\text { phase }\end{array}$ & Cockpit \\
\hline
\end{tabular}

Table 7

Summary table of the findings of focus 2 regarding the educational focus.

\begin{tabular}{|c|c|}
\hline Findings focus 2 & Supportive data \\
\hline $\begin{array}{l}\text { 2.I. Teachers and students consider that integrating formal and informal activities into the same learning setting } \\
\text { enriches the experience. }\end{array}$ & $\begin{array}{l}\text { [StudentsQuest] } \\
\text { [TeachersQuest] }\end{array}$ \\
\hline $\begin{array}{l}\text { 2.II. Students consider that the activity was useful to learn about the campus services and to locate and orientate around the buildings. Most of } \\
\text { them would recommend the activity and would repeat it for learning new things. }\end{array}$ & [StudentsQuest] \\
\hline 2.III. Students consider working in groups useful for sharing opinions, answering the questions, and meeting new people. & [StudentsQuest] \\
\hline \multirow[t]{2}{*}{ 2.IV. Teachers and students consider the activity as motivating. } & [StudentsQuest] \\
\hline & $\begin{array}{l}\text { [TeachersQuest] } \\
\text { [Interview] }\end{array}$ \\
\hline
\end{tabular}


4.3.2. Focus 2: support of the CSCBL script for the acquisition of the expected learning objectives

Focus 2 is devoted to analyze whether the students achieved the expected learning outcomes. Therefore, we seek to understand if the experience is educationally equivalent to the previous one enacted in 2009 despite their differences.

The first finding (2.I in Table 7, related to RQ5) shows that both teachers and students agree with that the integration of formal and informal activities into the same learning setting enriches the whole experience. Teachers qualify the experience as motivating for the students and highlight the successful way in which the different activities integrated the use of technology in students' daily life. One teacher says: "The integration serves to enrich the experience, because most of the students seemed quite motivated to do tasks that are, fundamentally, inane. Try asking a student to run to the library to figure out how the system works. Not exactly exciting stuff' [TeachersQuest]. Whilst students stress that combining activities help them to settle down regarding knowledge about the campus and learn more: "More activities=more benefits" [StudentsQuest]. Moreover, when students were asked whether they considered it more beneficial for learning doing the three activities together than doing them separately, only 2 students (over 32) gave a negative answer.

The second finding 2.II in Table 7 evidences that the CSCBL script successfully supported the learners' acquisition within their environment (RQ6). On the one hand, partial results show that students considered the experience useful to learn about the campus services, to know where to locate the different buildings and to orientate around the campus area. One of the students says: "(the activity is useful)... because the activity helps on discovering the campus" and another observes: "Because in a two-hour session we could learn where to locate everything in the campus" [StudentsQuest]. More specific examples show what students consider they learned from the experience: "(1) Location of the laboratories, (2) Library services and the (3) Location of the administrative university services' [StudentsQuest], “(1) Where the different buildings are, (2) how the library services work and (3) Other interesting services" [StudentsQuest], “(1) To learn about the campus, (2) To start meeting my course mates and (3) To know where the teachers" offices are located' [StudentsQuest]. A complete list of the answers is on the web data set http://mozart.gast.it.uc3m.es/ mmpsanag/escolab2010/.

Besides, when asked about three aspects that they learned from the experience most of them mentioned aspects related to the location of the different buildings and the services offered. These evidences are also supported by quantitative data. 16 (over 32) students answered that they would repeat the experience and, those who answered that they would not repeat the experience (15 over 32 ) is because they think they already knew enough about the campus. In one of the student's words: "(I would not repeat the experience because) I have enough information about the campus and its services" [StudentsQuest].

The third finding (2.III in Table 7, RQ7) shows how students perceived that the CSCBL script facilitated them to share opinions about their personal experiences helping them to meet new people. When asked which their preferred phase of the experiment was, half of the students selected the group activity because it was useful for meeting and building relationships with their new course mates. Moreover, some of the students that preferred the exploration with mobile phones phase also valued the fact of meeting new people: "Because we can meet the campus and new people at the same time" [StudentsQuest]. Finally, comments extracted from the answers about how students perceived the integration of the three activities into the same learning setting show that students value working in groups as very positive for sharing opinions and discussing with their mates.
Finally, the forth finding 2.IV in Table 7 (related to RQ8) evidences that the CSCBL script proposed fosters the students' motivation. On the one hand, teachers, although they have not participated in other similar activities, consider that these types of experiences motivate students, especially in more scientific courses. One of the teacher says: “(...) I think that this a very motivating activity for the students. Specially for particular areas of study" [Interview]. On the other hand, the number of students that would recommend the activity to their colleagues ( 30 over 32 ) as well as the adjectives they employ to describe the experience evidence their motivation on the experience. Some students qualify the activity as "innovative", "interesting" and "different" [StudentsQuest].

\section{Discussion and conclusions}

This paper has presented an in-depth analysis of the issues that appear when Computer Supported Collaborative Blended Learning scripts are deployed in real life scenarios. The work focused on how to take an already existing experience which was proven to be pedagogically positive, but expensive in terms of the time required by the teaching staff, and re-designing it to be deployed in a real life scenario with the support of a technology-based orchestration system.

We presented as a solution an orchestration system where the collaborative learning flow is managed and delivered to the participants by IMS Learning Design, and the activities are executed with the help of case specific tools and hardware. Generic Service Integration allows the adaptation of scripts based on the information obtained from these case specific tools, being such information exchanged with the IMS LD player during the enactment of the script. We also described a scenario in which these external services included mobile devices and Google Spreadsheets: the former were used by the students to interact with NFC tags, so that they received contextualized multimedia information of the environment; the latter were used to capture the data generated by the students and to create the students' groups. These applications allowed an intuitive and simple manipulation of numerous aspects of the activity flow.

We also presented an experiment. First, a detailed account of the limitations found regarding the enactment of the CSCBL script was documented. Second, we presented the requirements needed to overcome such limitations and the research questions addressed. The analysis of these research questions determined whether or not the requirements were fulfilled by the orchestration system. We considered several data sources for the mixed method adopted for the analysis. The experimental results sustain that the orchestration system properly provided guidance to the participants without restraining the flexibility of the script. Furthermore, the results also indicate that using IMS LD effectively supported the delivery of adaptive learning material and increased the reusability of the script. In summary, the system was able to enact the CSCBL script while keeping its cost, in terms of management tasks, at a minimum.

Another finding is that the enactment of the script with the support of the proposed orchestration system was verified to be similar to the original script version, in terms of learning benefits. More specifically, the analysis of the acquired learning outcomes reveals that: (i) the integration of formal and informal activities in the same flow enriched the whole experience, (ii) the CSCBL script properly supported the learners within the environment, and (iii) the script facilitated the exchange of personal views.

As a conclusion, in this paper we have presented a CSCBL script and a flexible architecture to support its enactment. On the one hand, we have shown that Generic Service Integration (GSI) enhances the flow-management capabilities of IMS Learning 
Design. The orchestration system offers a framework that keeps management tasks at a minimum and increases the reusability of the script, while maintaining the effectiveness of a learning experience even in challenging CSCBL scripts. The generalization of the presented case of study is based on the script division into macro and micro levels, where the former level is supported by a flow manager and the latter by case-specific tools, being these two levels connected by GSI. The underlying architecture that emerges from this particular case study contributes to the support of collaborative activities across different spatial locations. On the other hand, the results show that the architecture proposed dealt successfully with flexibility issues related to the group management typically related to the enactment of collaborative scripts.

Altogether might inspire other researchers to provide particularizations of this architecture to be used in other similar learning settings. Also, the CSCBL script design proposed can be adapted by any researcher and practitioner since it follows the structure defined by the 4SPPIces model [12]. The same design could be employed and re-adapted in other exploratory type of activities such as [46] in which students learn in a urban experience about the city of Barcelona. However, one of the difficulties to be addressed in this particularization process is to formalize the CSCBL script using IMS LD. Future work should facilitate this step with the creation of script templates and the provision of template based authoring tools such as Collage [33].

\section{Acknowledgments}

Work partially funded by the Learn3 project, "Plan Nacional de I+D+I" TIN2008-05163/TSI, the "Emadrid: Investigación y desarrollo de tecnologías para el e-learning en la Comunidad de Madrid" project (S2009/TIC-1650) and the EEE: Orquestando Espacios Educativos Especulares, "Plan Nacional de I+D+I" TIN2011-28308C03-03.

\section{References}

[1] H. Alavi, P. Dillenbourg, F. Kaplan, Distributed awareness for class orchestration, Learning in the Synergy of Multiple Disciplines (2009) 211-225.

[2] N. Nova, F. Girardin, P. Dillenbourg, 'Location is not enough!': an empirical study of location-awareness in mobile collaboration, in: IEEE International Workshop on Wireless and Mobile Technologies in Education, 2005, WMTE 2005, IEEE, 2006, pp. 21-28.

[3] G. Schwabe, C. Göth, Mobile learning with a mobile game: design and motivational effects, Journal of Computer Assisted Learning 21 (3) (2005) 204-216.

[4] G. Zurita, M. Nussbaum, Computer supported collaborative learning using wirelessly interconnected handheld computers, Computers \&amp; Education 42 (3) (2004) 289-314.

[5] M. Ruchter, B. Klar, W. Geiger, Comparing the effects of mobile computers and traditional approaches in environmental education, Computers \&amp; Education 54 (4) (2010) 1054-1067.

[6] R. Joiner, D. Stanton, J. Reid, R. Hull, D. Kirk, Facer, Savannah: mobile gaming and learning? Journal of Computer Assisted Learning 20 (6) (2004) 399-409.

[7] J. Moon, How to improve the impact of short courses and workshops, in: Training and Professional Development, Kogan Page, 2001.

[8] P. Dillenbourg, F. Fischer, Basics of computer-supported collaborative learning, Zeitschrift für Berufs-und Wirtschaftspädagogik 21 (2007) 111-130.

[9] P. Dillenbourg, F. Hong, The mechanics of CSCL macro scripts, International Journal of Computer-Supported Collaborative Learning 3 (1) (2008) $5-23$.

[10] G. Stahl, Group cognition in computer-assisted collaborative learning, Journal of Computer Assisted Learning 21 (2) (2005) 79-90.

[11] P. Dillenbourg, P. Tchounikine, Flexibility in macro-scripts for computersupported collaborative learning, Journal of Computer Assisted Learning 23 (1) (2007) 1-13.

[12] M. Pérez-Sanagustín, D. Hernández-Leo, P. Santos, J. Blat, 4SPPIces: a case study of factors in a scripted collaborative-learning blended course across spatial locations, International Journal of Computer Supported Collaborative Learning 7 (3) (2012) 443-465.
[13] A. Berger, R. Moretti, P. Chastonay, P. Dillenbourg, A. Bchir, R. Baddoura, C. Bengondo, D. Scherly, P. Ndumbe, P. Farah, et al. Teaching community health by exploiting international socio-cultural and economical differences, in: A. Eurelings, K. Hakkarainen, P. Dillenbourg (Eds.), Proceedings of the First European Conference on Computer Supported Collaborative Learning, 2001, pp. 97-105.

[14] P. Dillenbourg, P. Jermann, Designing integrative scripts, Scripting ComputerSupported Collaborative Learning (2007) 275-301.

[15] P. Jermann, P. Dillenbourg, Elaborating new arguments through a CSCL scenario, in: M. Baker, D. Suthers, J. Andriessen (Eds.), Arguing to Learn: Confronting Cognitions in Computer-Supported Collaborative Learning Environments, in: CSCL Series, vol. 1, 2003, pp. 205-226.

[16] J. Park, D. Parsons, H. Ryu, To flow and not to freeze: applying flow experience to mobile learning, IEEE Transactions on Learning Technologies 3 (1) (2010) 56-67.

[17] M. Pérez-Sanagustín, G. Ramírez-González, D. Hernández-Leo, M. Muñoz Organero, P. Santos, J. Blat, C. Delgado Kloos, Discovering the campus together: a mobile and computer-based learning experience, Journal of Network and Computer Applications 35 (1) (2012) 176-188.

[18] S. Ortiz Jr, Is near-field communication close to success? Computer 39 (3) (2006) 18-20.

[19] D. Hernández-Leo, J.I. Asensio-Pérez, Y. Dimitriadis, Computational representation of collaborative learning flow patterns using IMS learning design, Educational Technology \& Society 8 (4) (2005) 75-89.

[20] L. de-la-Fuente-Valentín, M. Pérez-Sanagustín, P. Santos, D. Hernández-Leo A. Pardo, C. Delgado Kloos, J. Blat, System orchestration support for a flow of blended collaborative activities, in: 2nd International Workshop on Adaptive Systems for Collaborative Learning, Thessaloniki, Greece, 2010, pp. 415-420.

[21] M. Bhuptani, S. Moradpour, RFID Field Guide: Deploying Radio Frequency Identification Systems, Prentice Hall PTR, Upper Saddle River, NJ, USA, 2005.

[22] L. de-la-Fuente-Valentín, Orchestration of learning activities through the integration of third-party services in IMS Learning Design. Ph.D. Thesis, Universidad Carlos III de Madrid, 2011.

23] M. Muñoz Organero, G.A. Ramírez-González, P.J. Muñoz Merino, C. Delgado Kloos, A collaborative recommender system based on space-time similarities, IEEE Pervasive Computing 9 (3) (2010) 81-87.

[24] T. Navarrete, P. Santos, D. Hernández-Leo, J. Blat, Qtimaps: a model to enable web maps in assessment, Educational Technology \& Society 14 (3) (2011) 203-217.

[25] IMS Learning Design specification. http://www.imsglobal.org/learningdesign/, Feb. 2003. Last visited April 2009.

[26] J.P. Escobedo Del Cid, L. de-la-Fuente-Valentín, S. Gutiérrez, A. Pardo, C. Delgado Kloos, Implementation of a learning design run-time environment for the IRN learning management system, Journal of Interactive Media in Education Special Issue: Adaptation and IMS Learning Design (2007) 12.

[27] L. de-la-Fuente-Valentín, A. Pardo, C. Delgado Kloos, Generic service integration in adaptive learning experiences using IMS learning design, Computers \& Education 57 (1) (2011) 1160-1170.

[28] C. Delgado Kloos, A. Pardo, M. Muñoz-Organero, M.B. Ibáñez, R. Crespo, P. Muñoz Merino, L. de-la-Fuente-Valentín, D. Leony, I. Gutiérrez, Some research questions and results of UC3M in the eMadrid excellence network. in: EDUCON 2010 IEEE Annual Global Engineering Education Conference, Madrid, 2010, pp. 1101-1110.

[29] P. Laforcade, A Domain-Specific modeling approach for supporting the specification of visual instructional design languages and the building of dedicated editors, Journal of Visual Languages \& Computing (2010).

[30] A. Lejeune, M. Ney, A. Weinberger, M. Pedaste, L. Bollen, T. Hovardas, U. Hoppe Ton De Jong, Learning activity spaces: towards flexibility in learning design? in: International Conference on Advanced Learning Technologies, 2009, pp. 433-437.

[31] B. Fernández-Gallego, M. Lama, J.C. Vidal, E. Sánchez, A. Bugarín, OPENET4VE: a platform for the execution of IMS LD units of learning in virtual environments, in: IEEE International Conference on Advanced Learning Technologies, IEEE Computer Society, 2010, pp. 472-474.

[32] I. Martinez-Ortiz, J.L. Sierra, B. Fernandez-Manjon, Authoring and reengineering of IMS learning design units of learning, IEEE Transactions on Learning Technologies 2 (3) (2009) 189-202.

[33] D. Hernández-Leo, E. Villasclaras-Fernández, I.M. Jorrín-Abellán, J.I. AsensioPérez, Y. Dimitriadis, I. Ruiz-Requies, B. Rubia-Avi, COLLAGE, a collaborative learning design editor based on patterns, Technology \& Society 9 (1) (2006) $58-71$.

[34] D. Burgos, C. Tattersall, R. Koper, How to represent adaptation in e-learning with IMS-learning design, Interactive Learning Environments 15 (2) (2007) 161-170.

[35] S. Neumann, M. Klebl, D. Griffiths, L. de-la-Fuente-Valentín, D. HernándezLeo, H. Hummel, F. Brouns, M. Derntl, P. Oberhuemer, Report of the results of an IMS learning design expert workshop, International Journal of Emerging Technologies 5 (1) (2010) 58-72.

[36] L. Palomino-Ramírez, M. Bote-Lorenzo, J.I. Asensio-Perez, Y. Dimitriadis, L. de-la-Fuente-Valentín. The data flow problem in learning design: a case study, in: Proceedings of the International Conference on Networked Learning, Halkidiki, Greece, May 2008, pp. 285-292. 
[37] A. Turani, R. Calvo, Sharing synchronous collaborative learning structures using IMS learning design, in: 2006 7th International Conference on Information Technology Based Higher Education and Training, IEEE, 2006, pp. 25-34.

[38] V. Monfort, M. Khemaja, S. Hammoudi, Extending the IMS LD standard with adaptability, in: IEEE International Conference on Advanced Learning Technologies, IEEE Computer Society, 2010, pp. 320-322.

[39] M. Khemaja, S. Ghallabi, V. Monfort, Towards intelligent collaborative learning simulations: extending the IMS LD standard by web semantic based ITSs, in: IEEE International Conference on Advanced Learning Technologies, IEEE Computer Society, 2010, pp. 642-643.

[40] R. Valdivia, M. Nussbaum, S.F. Ochoa, Modeling a collaborative answer negotiation activity using IMS-based learning design, IEEE Transactions on Education 52 (3) (2009) 375-384.

[41] R. García-Robles, F. Díaz-del Rio, S. Vicente-Díaz, An eLearning standard approach for supporting PBL in computer engineering, IEEE Transactions on Education 52 (3) (2009) 328-339.

[42] L. de-la-Fuente-Valentín, A. Pardo, C. Delgado Kloos, Generic service integration in adaptive learning experiences using IMS learning design, Computers \& Education 57 (1) (2011) 1160-1170.

[43] A. Martínez, Y. Dimitriadis, B. Rubia, E. Gómez, P. De La Fuente, Combining qualitative evaluation and social network analysis for the study of classroom social interactions, Computers \& Education 41 (4) (2003) 353-368.

[44] R. Burke Johnson, L. Christensen, Educational Research: Quantitative, Qualitative, and Mixed Approaches, Sage Publications, Inc., 2007.

[45] L. de-la-Fuente-Valentín, A. Pardo, J.I. Asensio Pérez, Y. Dimitriadis, C. Delgado Kloos, Collaborative learning models on distance scenarios with learning design: a case study. in: International Conference on Advanced Learning Technologies, Santander, Spain, 2008, pp. 278-282.

[46] P. Santos, M. Pérez-Sanagustín, D. Hernández-Leo, J. Blat, QuesTInSitu: from tests to routes for assessment in situ activities, Computers \&amp; Education 57 (4) (2011) 2517-2534. 